\title{
Validación factorial de un cuestionario para medir la conducta de comer en ausencia de hambre y su asociación con obesidad infantil
}

\author{
PAMELA MORALES M. ${ }^{1}$, JOSÉ LUIS SANTOS M. ${ }^{2}$, ANDREA GONZÁLEZ S. ${ }^{3}$, \\ JUDITH HO U. ${ }^{3}$, MARÍA ISABEL HODGSON B. ${ }^{4}$ \\ 1. Residente de Pediatría, División de Pediatría, Pontificia Universidad Católica de Chile. \\ 2. Bioquímico. PhD, Laboratorio de Nutrición y Genética, Departamento de Nutrición, Diabetes y Metabolismo. \\ Escuela de Medicina. Pontificia Universidad Católica de Chile. \\ 3. Alumno Magister Nutrición. Departamento de Nutrición, Diabetes y Metabolismo. Escuela de Medicina. \\ Pontificia Universidad Católica de Chile. \\ 4. Nutrióloga Infantil. División de Pediatría y Departamento de Nutrición, Diabetes y Metabolismo. \\ Escuela de Medicina. Pontificia Universidad Católica de Chile.
}

\begin{abstract}
A questionnaire to measure eating behavior in the absence of hunger and its association with childhood obesity

Background: It has been observed that some psychological factors tend to stimulate food intake in the absence of hunger in obese children. Objective: To evaluate whether obese children have a greater tendency to eat in the absence of hunger, in response to various emotional and environmental factors versus normal weight children. Subjects and Methods: Obese patients were selected according to NCHS/CDC2000 ( $\mathrm{n}=49$ and $\mathrm{n}=99$ for nonobese children), males and females in the age group of 6-12 years who consulted in the UC health network and also children that were evaluated in schools located in southeastern Santiago. The questionnaire "Eating in the absence of hunger" was used, which measures three subscales: external stimuli, fatigue/boredom and negative feelings. Results: Factor analysis showed a significant degree of overlap between the "fatigue/boredom" and "negative feelings" subscales. Obese children had higher scores on "external stimuli" versus normal weight children (median 2.7 compared to 1.7, $\mathrm{p}<0.001$ ). In the "fatigue/boredom" subscale, scores of 2.5 versus 1.2 ( $\mathrm{p}$ $<0.001)$ were obtained, while in "negative feelings", scores reported 2.0 versus $1.2(p=0.0013)$. Conclusions: Obese patients reported higher scores on the questionnaire "Eating in the absence of hunger" than non-obese children, identifying modifiable and educable stimuli that could prevent this eating behavior.

Key words: (Obesity, eating in the absence of hunger, eating behavior).

Rev Chil Pediatr 2012; 83 (5): 431-437
\end{abstract}

Recibido el 03 de marzo de 2011, devuelto para corregir el 16 de mayo de 2011, segunda versión el 10 de enero de 2012 , tercera versión el 26 de abril de 2012, aceptado para publicación el 01 de mayo de 2012.

Este trabajo cumple con los requisitos sobre consentimiento /asentimiento informado, comité de ética, financiamiento, estudios animales y sobre la ausencia de conflictos de intereses según corresponda.

Correspondencia a:

María Isabel Hodgson B.

E-mail: hodgson@med.puc.cl 


\section{RESUMEN}

Introducción: Se ha observado que en los niños obesos, algunos factores psicológicos tienden a estimular la ingesta de alimentos en ausencia de hambre. Objetivo: Evaluar si los niños obesos presentan mayor tendencia a comer en ausencia de hambre, en respuesta a distintos factores emocionales y ambientales, en comparación con niños normopeso. Pacientes y Método: Se seleccionaron pacientes obesos según NCHS/CDC2000 (n= 49) y normopeso $(n=99)$ de ambos sexos y con edades entre 6-12 años, que consultaron en forma espontánea en la Red de Salud UC y niños evaluados en colegios del sector suroriente de Santiago. Se aplicó el cuestionario "Comer en ausencia de hambre" que mide tres subescalas: Estímulos externos, cansancio/aburrimiento y sentimientos negativos. Resultados: El análisis factorial reveló un importante grado de solapamiento entre las ponderaciones de las subescalas "cansancio/aburrimiento" y "sentimientos negativos". Los niños con obesidad presentaron mayores puntajes en la dimensión "estímulos externos" que los niños normopeso (mediana de 2,7 versus 1,7; $\mathrm{p}<0,001$ ). En "cansancio/aburrimiento" se obtuvieron puntajes de 2,5 versus $1,2(\mathrm{p}<0,001)$ mientras que en "sentimientos negativos" los puntajes fueron 2,0 versus $1,2(\mathrm{p}=0,0013)$. Conclusiones: Los pacientes obesos presentaron mayores puntajes en la encuesta alimentaria "Comer en ausencia de hambre" que los niños normopeso, pudiendo identificarse estímulos modificables y educables que podrían prevenir esta conducta alimentaria.

(Palabras clave: Obesidad, comer en ausencia de hambre, conducta alimentaria).

Rev Chil Pediatr 2012; 83 (5): 431-437

\section{Introducción}

La obesidad es un desorden multifactorial en dónde interactúan factores genéticos y ambientales que operan a nivel celular, tisular, fisiológico y social ${ }^{1}$. Se ha estudiado el comportamiento alimentario con múltiples encuestas, observándose que los niños obesos tienen menor respuesta a señales internas relacionadas con la saciedad, tienden a comer más rápido durante las comidas y son más sensibles a los estímulos externos relacionados con los alimentos ${ }^{2,3}$.

Existen niños que comienzan a comer en ausencia de hambre fisiológica, o bien continúan comiendo pese a no sentir hambre. También se ha observado que algunos factores psicológicos como el estrés, el ánimo bajo, el aburrimiento y la soledad, son capaces de estimular la ingesta alimentaria en ausencia de hambre en niños obesos. Para tratar de investigar la influencia de estos factores en el acto de comer se desarrolló el cuestionario "Eating in The Abscence of Hunger" (EAH-C), en el cual se busca evaluar la influencia de estados emocionales tales como: tristeza o depresión; enojo o frustración; ansiedad o nerviosismo; cansancio y aburrimiento, y el efecto de algunos precipitantes externos, ya sea estimulos sensoriales (ver comida, sentir sabores u olores) o estímulos sociales (ver a otras personas comiendo), en el inicio o continuación de una comida ${ }^{4}$.
El cuestionario "EAH-C", adaptado para niños y adolescentes considera tres dimensiones: a) comer en respuesta a emociones negativas; b) comer en respuesta a factores externos; y c) comer en respuesta a aburrimiento ${ }^{4}$.

Se ha observado que madres que presentan conductas que favorecen la alimentación en ausencia de hambre, tienen hijas que también presentan este comportamiento, ya sea porque existe un componente genético o un comportamiento aprendido, aumentando el riesgo de sobrepeso y obesidad en las niñas que lo presentan ${ }^{5}$.

El objetivo del presente estudio es evaluar si los niños obesos presentan mayor tendencia a comer en ausencia de hambre, en respuesta a distintos factores emocionales y ambientales, en comparación con niños normopeso.

Nuestra hipótesis es que los niños chilenos obesos presentan con mayor frecuencia la conducta alimentaria de comer en ausencia de hambre, en respuesta a distintos factores emocionales y externos, en comparación con niños normopeso.

\section{Pacientes y Método}

\section{Sujetos}

Se reclutaron niños entre 6 y 12 años, considerándose obesos los que presentaban índice de masa corporal (IMC) mayor del percentil 95 
y normopeso (controles) a aquellos con IMC entre p25-75 según estándares de referencia NCHS/CDC $2000^{6}$, los niños fueron reclutados mediante consulta espontánea con nutrióloga y/o pediatra general en la Red de Salud de la Universidad Católica (UC) y en establecimientos educacionales del sector Suroriente de la Región Metropolitana. Se consideraron criterios de exclusión, la presencia de comorbilidades endocrinológicas diagnosticadas previamente y aquellos niños con diagnóstico previo de obesidad que hubiesen recibido alguna intervención nutricional previa al estudio.

El tamaño de la muestra considerado en este estudio se calculó basándose en las diferencias en puntajes de conducta de alimentación de similar magnitud a las encontradas en el trabajo de Santos et $\mathrm{al}^{3}$. El tamaño de muestra obtenido entrega un poder estadístico de $90 \%$ para encontrar diferencias significativas entre grupos obesos y no-obeso, con una probabilidad de error de tipo 1 menor a 0,05 .

En ambos grupos se realizó antropometría con medición de peso (medido en balanza Seca con precisión de 100 gramos), talla (medido en estadímetro Seca con precisión de 1 centímetro) y circunferencia abdominal (medido con cinta métrica inextensible con precisión de $1 \mathrm{~mm}$ ). El desarrollo puberal fue evaluado según criterios de Tanner, por 3 evaluadores entrenados ${ }^{7}$.

El estudio fue aprobado por el comité de ética de la Facultad de Medicina de la Pontificia Universidad Católica de Chile.

\section{Cuestionario "Comer en ausencia de hambre"}

Se aplicó el cuestionario: "EAH-C", previamente adaptado para niños y adolescentes, con propiedades psicométricas evaluadas por los autores del cuestionario ${ }^{4}$. Dicha encuesta fue solicitada en forma directa a sus autores, quienes autorizaron su aplicación en Chile. El cuestionario original fue adaptado a la población chilena mediante traducción directa e inversa y fue aplicado a las madres de los niños reclutados, por 3 encuestadores entrenados $^{4}$. El cuestionario está compuesto por 14 ítems que evalúan tres dimensiones relacionadas con los estímulos que generan la ingesta de alimentos en ausencia de hambre: a) Sen- timientos negativos: inicio o mantención de la ingesta alimentaria en relación a tristeza, ira o ansiedad; b) Estímulos externos: inicio o mantención de ingesta de alimentos desencadenada por ver comida o que alguien más se encuentre comiendo en el entorno y c) Cansancio y aburrimiento: inicio o mantención de la ingesta de alimentos estimulada por la presencia de estas situaciones. Las tres dimensiones se evalúan en dos contextos distintos, como son, comer inmediatamente después de una comida o comer dos horas después de una comida.

\section{Análisis factorial confirmatorio}

Sobre el conjunto de las respuestas al cuestionario, se aplicaron técnicas de análisis factorial con método de extracción de componentes principales y rotación ortogonal de ejes (rotación varimax) (comando "factor" en STATA 12, opción “pcf”; y comando "rótate"). El objetivo de este análisis es identificar la presencia de un reducido número de factores que expliquen la variabilidad de los datos, así como confirmar la estructura de factores del cuestionario original ${ }^{8}$.

\section{Análisis de la consistencia interna}

Se calcularon los valores de Cronbach's $\alpha$ para evaluar la consistencia interna de las distintas dimensiones evaluadas.

\section{Validación de la subescala de conducta de alimentación por "estímulos externos"}

En un estudio paralelo se realizó una prueba piloto en 10 niños normopeso ( 8 de ellos varones) a los cuales se aplicó el cuestionario EAH-C. Posteriormente y bajo condiciones controladas, se ofreció a los niños una comida estandarizada y se los instruyó para que comieran hasta que se sintieran saciados. Una vez finalizada esta comida de precarga, se ubicó a los niños en salas individuales y se les entregó un juego de salón y un paquete prepesado con diferentes snacks dulces para que comieran mientras jugaban. Luego de 15 minutos se retiraron los paquetes para pesar el remanente y calcular la cantidad de alimentos dulces consumidos en la "prueba de comer en ausencia de hambre", expresada en gramos y calorías según el etiquetado nutricional de 
cada producto. Estos resultados se correlacionaron con los puntajes obtenidos en el cuestionario inicial.

\section{Métodos estadísticos del estudio de caso-control}

La comparación de casos (GO) y controles (GC) se analizó mediante pruebas estadísticas de variables continuas no paramétricas (test de Mann Whithney). Todos los análisis estadísticos se realizaron mediante el programa STATA 11.0.

\section{Resultados}

Se reclutaron 148 niños entre 6-12 años, 49 niños con IMC mayor del p95 y 99 niños normopeso con IMC entre p 25$75^{5}$, los niños fueron reclutados mediante consulta espontánea con nutrióloga y pediatra general en la Red de Salud de la Universidad Católica y en establecimientos educacionales en el sector suroriente de la Región Metropolitana. En el grupo de casos y controles $53,1 \%$ y $45,5 \%$ correspondían a mujeres respectivamente, no presentando diferencia significativa entre ambos grupos. El promedio de edad fue de 9,6 $\pm 2,1$ años en el GC y de $11 \pm 1,2$ años en el GO. Ambos grupos presentaron diferencia significativa en IMC y circunferencia abdominal, como era esperable (tabla 1).

\section{Análisis factorial}

El análisis factorial identificó una solución de tres factores con valores propios (eigenvalues) superiores a 1, que explican hasta un $71,3 \%$ de la varianza total de los datos. En el análisis de ponderaciones de cada ítem tras la rotación ortogonal, se observó que los "estímulos externos" están claramente definidos como un factor independiente, mientras que existe un importante grado de solapamiento entre las ponderaciones de los items de los factores 2 y 3 , que corresponderían a las dimensiones de "sentimientos negativos" y "cansancioaburrimiento". Sin embargo, con el objeto de mantener la comparabilidad con otros
Tabla 1. Características generales y antropométricas de los grupos de niños obesos y normopeso

\begin{tabular}{|lccc|}
\hline & $\begin{array}{c}\text { Obesos } \\
(\mathbf{n}=\mathbf{4 9})\end{array}$ & $\begin{array}{c}\text { Normopeso } \\
(\mathbf{n}=\mathbf{9 9})\end{array}$ & P \\
\hline Edad (años) & $9,6 \pm 2,1$ & $11,0 \pm 1,2$ & NS \\
\hline $\begin{array}{l}\text { Sexo (\% niñas) } \\
\text { Desarrollo puberal } \\
\text { (\% Tanner 1-2) }\end{array}$ & 53,1 & 45,5 & NS \\
\hline IMC (kg/m $\left.{ }^{2}\right)$ & $25,6 \pm 3,5$ & $16,9 \pm 1,8$ & $<0,001$ \\
\hline Z score IMC & $3,60 \pm 1,55$ & $-0,07 \pm 0,66$ & $<0,001$ \\
\hline Talla (cm) & $142,0 \pm 12,3$ & $141,8 \pm 7,8$ & NS \\
\hline $\begin{array}{l}\text { Perímetro abdominal } \\
\text { (cm) }\end{array}$ & $87,1 \pm 10,8$ & $63,9 \pm 6,2$ & $<0,001$ \\
\hline Vaíable cuantitativas & & 67,6 & NS \\
\hline
\end{tabular}

Variables cuantitativas expresadas como media \pm desviación estándar. IMC: Indice de masa corporal, cm: centímetros, kg: kilogramos, m: metro.

Tabla 2. Análisis de la estructura de factores del cuestionario: "Comer en ausencia de hambre"

\begin{tabular}{|c|c|c|}
\hline $\begin{array}{l}\text { Número } \\
\text { de Factor }\end{array}$ & $\begin{array}{l}\text { Preguntas: } \\
\text { ¿Con qué frecuencia }\end{array}$ & $\begin{array}{l}\text { Ponderación } \\
\text { factorial }\end{array}$ \\
\hline \multirow{6}{*}{$\begin{array}{l}\text { Factor } 1 \\
54,4 \%\end{array}$} & Continúa comiendo si & \\
\hline & ... se siente aburrido? & 0,77 \\
\hline & ... se siente ansioso o nervioso? & 0,78 \\
\hline & Empieza a comer si... & \\
\hline & ... se siente aburrido? & 0,73 \\
\hline & ... se siente ansioso o nervioso? & 0,80 \\
\hline \multirow{8}{*}{$\begin{array}{l}\text { Factor } 2 \\
9,1 \%\end{array}$} & Continúa comiendo si & \\
\hline & ... se siente triste o deprimido? & 0,75 \\
\hline & ... se siente enojado o frustrado? & 0,67 \\
\hline & ... se siente cansado? & 0,79 \\
\hline & Empieza a comer si... & \\
\hline & ... se siente triste o deprimido? & 0,53 \\
\hline & ... se siente enojado o frustrado? & 0,66 \\
\hline & ... se siente cansado? & 0,79 \\
\hline \multirow{6}{*}{$\begin{array}{l}\text { Factor } 3 \\
7,7 \%\end{array}$} & Continúa comiendo si & \\
\hline & $\begin{array}{l}\text {.. la comida se ve, sabe o huele } \\
\text { muy bien? }\end{array}$ & 0,52 \\
\hline & los demás siguen comiendo? & 0,55 \\
\hline & Empieza a comer si... & \\
\hline & $\begin{array}{l}\text {... la comida se ve, sabe o huele } \\
\text { muy bien? }\end{array}$ & 0,79 \\
\hline & los demás siguen comiendo? & 0,79 \\
\hline
\end{tabular}


estudios, se realizó el análisis con los tres factores separadamente ${ }^{4}$. Adicionalmente, se analizaron los datos utilizando un nuevo factor generado por la combinación de la información de "sentimientos negativos" y "cansancio-aburrimiento", al que hemos llamado "contextos negativos"(tabla 2 ).

\section{Consistencia interna}

Se evaluó la consistencia interna mediante la estadística Cronbach's $\alpha$ siendo para la dimensión sentimientos negativos de 0,90 , para factores externos de 0,81 , cansancio y aburrimiento de 0,79 . La nueva dimensión contextos negativos presentó un Cronbach's $\alpha$ de 0,92.
Se considera generalmente una buena consistencia interna con Cronbach's $\alpha$ mayor a $0,75^{9}$.

\section{Validación de la subescala de conducta de alimentación por "estímulos externos"}

En la prueba piloto realizada se observó que existe una buena correlación entre la ingesta en ausencia de hambre incentivada por estímulos externos y el puntaje obtenido en el cuestionario "comer en ausencia de hambre", aplicado a los niños evaluados (figura 1).

\section{Caso-Control}

Al evaluar nuestro objetivo primario (figura 2) y comparar los puntajes obtenidos por los
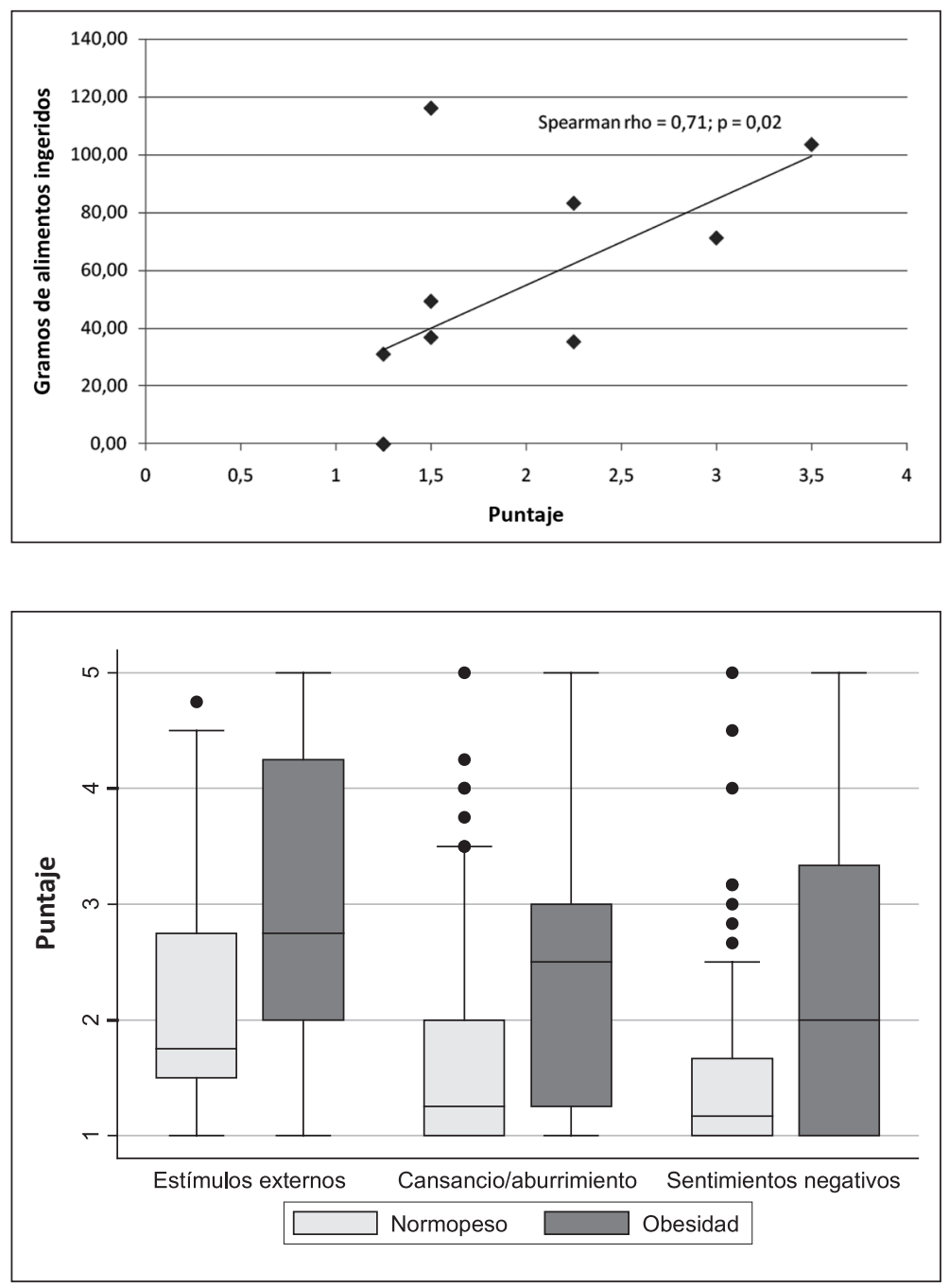

Figura 1. Validación de la subescala de conducta de alimentación por "estímulos externos". (1) En el eje de las abcisas se muestran los puntajes estandarizados de el cuestionario "comer en ausencia de hambre" y en el eje de las ordenadas la cantidad de alimentos ingeridos, en gramos. (2) Se observa una adecuada correlación entre el puntaje obtenido en el cuestionario y la cantidad de alimentos ingeridos.

Figura 2. Comparación de puntajes obtenidos en los grupos normopeso y obesos. (1) En el eje de las abscisas se representan los grupos de obesidad-normopeso en las diferentes dimensiones evaluadas. (2) En el eje de las ordenadas se representan los puntajes obtenidos estandarizados en cada dimensión en una escala del 1 al 5. (3) Se observa una diferencia significativa en las tres dimensiones evaluadas: estímulos externos $\mathrm{p}<0,001$, cansancio/aburrimiento $\mathrm{p}=0,0013$ y sentimientos negativos $\mathrm{p}<0,001$. 
dos grupos, se pudo observar que en la dimensión sentimientos negativos la mediana de puntaje obtenido por niños obesos fue de 2,0 por cada ítem en comparación con controles que obtuvieron 1,2 ( $p=0,0013)$. En la dimensión estímulos externos los obesos presentaron una mediana de 2,7 y los controles $1,7(\mathrm{p}<0,001)$ y en la dimensión de cansancio/ aburrimiento el grupo de obesos presentó una mediana de 2,5 y los controles $1,2(p<0,001)$. Se evaluó en forma independiente la nueva dimensión "contextos negativos" obteniéndose una mediana en obesos de 2,3 puntos en comparación con 1,4 observada en controles $(p<0,001)$.

$\mathrm{Al}$ analizar los grupos separados por sexo, los varones obesos presentaron puntajes más altos que los controles normopeso en todas las dimensiones evaluadas $(\mathrm{p}=0,002 ; \mathrm{p}=0,001$; $\mathrm{p}<0,001)$. Las niñas obesas presentaron puntajes más altos en las dimensiones cansancio/ aburrimiento, factores externos y contextos negativos $(\mathrm{p}=0,004 ; \mathrm{p}=0,001$ y $\mathrm{p}=0,005$ respectivamente), no así en la dimensión de sentimientos negativos $(\mathrm{p}=0,11)$.

\section{Discusión}

A pesar de las estrategias implementadas para tratar de revertir el avance de la obesidad, la prevalencia de ésta en población escolar sigue mostrando un ascenso progresivo en los últimos años ${ }^{10}$. Esto motiva la búsqueda de factores modificables que puedan frenar su avance o contribuir a su tratamiento.

Nuestro estudio muestra que los niños obesos entre 6 y 12 años, presentan puntajes mayores en todos los parámetros incluidos en el cuestionario "EAH-C" en comparación a los niños controles normopeso. Resultados similares se han descrito en estudios extranjeros pre$\operatorname{vios}^{5,8,11}$. El comer sin hambre se observa en presencia de los distintos estímulos evaluados; emocionales y ambientales.

En nuestro estudio, fue posible evaluar los estímulos que pueden influenciar la conducta alimentaria de comer en ausencia de hambre. Hemos observado que estímulos modificables como el aburrimiento, estímulos relacionados con la disponibilidad de comida o el ver a otras personas del entorno comiendo, ya sea en la casa o en el colegio, pueden estimular la ingesta de alimentos en ausencia de hambre. Esta conducta puede dificultar el tratamiento de la obesidad si no se modifica el contexto familiar y social, especialmente en lo que se refiere al control de los factores externos que estimulan o desencadenan el acto de comer en ausencia de hambre.

Al realizar el análisis de la estructura de factores pudimos observar que, a diferencia del cuestionario original de Tanosfsky, dos de éstos presentan un solapamiento en la estructura de factores entre las preguntas que corresponden a las dimensiones de "Sentimientos negativos" y "Cansancio/aburrimiento", originalmente descritas en el cuestionario. La unión de ambas dimensiones da origen a una nueva dimensión que hemos denominado "contextos negativos" . Por lo tanto, en nuestro estudio, los factores que influyen en el comer sin hambre podrían resumirse en dos aspectos fundamentales, aquellos propios del individuo, dependientes de su estado anímico y psicológico, y aquellos dependientes del entorno familiar y social. Ambos necesitan ser abordados con estrategias diferentes al enfrentar la evaluación y tratamiento de un niño obeso, y también deben ser tomados en consideración si se pretende avanzar en programas de prevención de sobrepeso y obesidad. Por otra parte, podemos afirmar que las dimensiones básicas del cuestionario se replicaron satisfactoriamente en niños chilenos.

Como una limitación de nuestro estudio, se puede establecer que a pesar de que el tamaño muestral entrega un poder estadístico adecuado para el grupo etario específico en el cual fue realizado el cuestionario, éste no puede considerarse representativo de la población pediátrica general.

En conclusión, los pacientes obesos comen más en ausencia de hambre que los niños normopeso, lo que está determinado por distintos estados emocionales y estímulos ambientales/ sociales. Por último, es importante destacar que nuestro estudio identifica factores modificables y prevenibles que estimulan esta conducta, cuyo manejo puede constituir una indicación médica en la consulta pediátrica habitual, que permita abordar con mayor eficacia el tratamiento de los niños obesos. 


\section{Agradecimientos}

Queremos expresar nuestro agradecimiento al Dr. Marian Tanofsky por facilitarnos la encuesta para realizar este estudio.

\section{Referencias}

1.- Webber L, Hill C, Saxton J, Van Jaarsveld CH, Wardle $J$ : Eating behaviour and weight in children. Int J Obes 2009; 33: 21-8.

2.- Domínguez Vásquez P, Olivares S, Santos JL: Influencia familiar sobre la conducta alimentaria y su relación con la obesidad infantil. Arch Latinoam Nutr 2008; 58: 24953.

3.- Santos JL, Ho-Urriola J, González A, et al: Association between eating behavior scores and obesity in Chilean children. Nutrition Journal 2011; 10: 108.

4.- Tanofsky-Kraff M, Ranzenhofer LM, Yanovski SZ, et $a l$ : Psychometric properties of a new questionnaire to assess eating in the absence of hunger in children and adolescents. Appetite 2008; 51: 148-55.
5.- Fisher JO, Birch LL: Eating in the absence of hunger and overweight in girls from 5 to $7 \mathrm{y}$ of age. Am J Clin Nutr 2002; 76: 226-31.

6.- Ogden CL, Kuczmarski RJ, Flegal KM, et al: Centers for Disease Control and Prevention 2000 growth charts for the United States: Improvements to the 1977 National Center for Health Statistics version. Pediatrics 2002; 109 (1): 45-60.

7.- Tanner JM: Growth and maturation during adolescence. Nutr Rev 1981; 39: 43-55.

8.- Sleddens EF, Kremers SP, Thijs C: The Children's Eating Behaviuor Questionnaire validity and association with Body Mass Index in Dutch children age 6-7. Int J Behav Nutr Phys Act 2008; 5: 49-58.

9.- Bland JM, Altman DG: Statistics notes: Cronbach's Alpha. BMJ 1997; 314: 572-3.

10. Situación nutricional de los escolares chilenos en primero básico http://venus.junaeb.c

11.- Faith MS, Berkowitz RI, Stallings VA, Kerns J, Storey $M$ and Stunkard AJ Eating in the absence of hunger: A genetic marker for childhood Obesity in Prepubertal Boys? Obesity 2006; 14: 131-8. 